\title{
The Silent Voice of Those Who are no Longer: Transgenerational Transmission of Information from the Perspective of the Informational Model of Consciousness
}

ISSN: 2578-0093

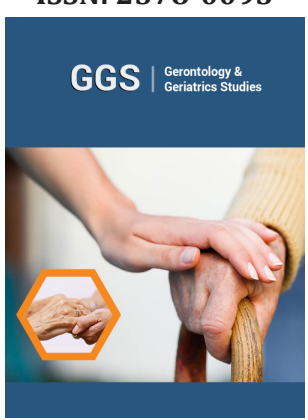

*Corresponding author: Florin Gaiseanu, Science of Information and Technology, Romania and Spain.

\section{Submission: 漈 August 14, 2019}

Published: 海 September 06, 2019

Volume 5 - Issue 1

How to cite this article: Florin Gaiseanu. The Silent Voice of Those Who are no Longer: Transgenerational Transmission of Information from the Perspective of the Informational Model of Consciousness. Gerontol \& Geriatric stud.5(1). GGS.000604.2019.

DOI: $10.31031 /$ GGS.2019.05.000604

Copyright@ Florin Gaiseanu, This article is distributed under the terms of the Creative Commons Attribution 4.0 International License, which permits unrestricted use and redistribution provided that the original author and source are credited.

\section{Florin Gaiseanu*}

Science of Information and Technology, Romania and Spain

\begin{abstract}
The "nature or nurture" problem concerning the debate on the innate features with respect to the acquired ones is approached in terms of information, from the perspective of the Informational Model of Consciousness. This model reveals seven distinct informational systems reflected in consciousness as informational centers, i.e. memory (Iknow-Ik), decisional info-operational center (Iwant-Iw), emotions (Ilove-Il), metabolic operations (Iam-Ia), genetic transmission (Icreate-Ic), genetic info-generation (Icreated-Icd) and the anti-entropic center (Ibelieve-Ib). Ib is a life-assisting beneficial center, because it is opposed to the entropic action of matter, eliminating or reducing the uncertainty characteristic of unknown possibilities to certainty, and inducing in this way the trust and confidence in own actions. The pro and contra arguments of the "nature or nurture" are shortly presented, noting the large range of divergent opinions and concepts on the approached problem. It is shown that the information concept acting both as informal and matter-related information, specifically referred to the embodiment/ disembodiment mechanisms for the transmission of information during the epigenetic processes, can coherently approach and explain the transgenerational transmission of the traits acquired during the life span of the predecessors. The concepts of tendency, affinity, propensity, predisposition, aptitude, vocation and talent, specific for inheritable properties described by Icd are discussed, pointing out the large pallet and variety of such concepts describing the evidences detected by external observers on the inherited behaviors, and that of self-perceived by the implicated individual, as a silent voice of whose are no longer. The external manifestation of such inherited features and traits depends on practice, because the characteristic operation of the nervous system and of epigenetic processes is based on repetition and/or intensive practice, but these are permanently guided by the silent voice of those who are no longer, with various signal intensities.
\end{abstract}

\section{Introduction}

Since a long time, probably since always, people did not wonder that the newborn and the future child resembled the mother, the father, the grandparents or even great-grandfather or great-grandmother, both as a physiognomy or behavioral style and preoccupations, because this seems to be a natural common situation. However, the science, starting with philosophy and continuing with branches such as psychology and psychiatry, the humanities and social sciences, as well as the behavioral and cognitive sciences, are still wondering since about 50 years, which is the appropriate answer to the "nature or nurture" problem. That means-in inheritability terms-what is determinant for the new generation: the inherited or the acquired properties during the span life? In other words, if the characteristics of a person in a certain field of activity, usually in music, in art in general, in mathematics or other sciences, in athletic or other performance fields requiring a distinct level of intelligence and/or dexterity, are the result of predispositions, innate aptitudes, of talent, or rather of the technical skills and of the exercise practiced with abnegation and tenacity [1]. For this purpose, the testing methods and especially the approaching mode of this problem were brought into discussion, both from a conceptual or interpretation point of view, and the balance between the two fundamental alternatives was discussed, but surprisingly, under the form of "all or nothing", which means, one excludes the other [1].

Could be this the right answer to such a fundamental question, defying sometimes even the evidences? We do not have yet enough research tools and knowledge to elucidate this 
"problem", intervening this time from the perspective of the modern sciences of information, of neurosciences and of genetics? As expected, a series of interventions to this surprising result, trying to restore, from various points of view, the balance lost in such an exclusionist conclusion, have outlined the followings aspects:

(i) It is not suitable to reduce the „nature - nurture” problem to only two extreme alternatives, this asking for instance to apply a asymptotic comparative analysis within so-called testing-the-limits method [2], or other elaborated models [3];

(ii) The identification and testing of the fundamental specific parameters of the human "processor", like working memory capacity, learning rates, and forgetting rates should be taken into account [4];

(iii) It is wrong to exclude the motivation and self-confidence in the personal training, because these comes from an intimate discovery of special sensitivity, and even worse, to reduce this problem to a ban-imposed egalitarianism ideology, justified by a false interpretation of the equality of opportunities [5];

(iv) It is absurd to ignore the heritability of musical talent, evoking only the "environmentalism" factor [6];

(v) The perseverance, the hard but gradual work and the industriousness cannot be underestimated [7]; to explain suitably the relation between innate talent and high levels of ability, it is necessary to consider the differences in structure between most everyday abilities and expert performance [8];

(vi) Practice and other factors are important contributors to outstanding performance, but not enough to explain great creative works [9];

(vii) Experience is no improvement over talent [10];

(viii) General intelligence is central to many forms of talent
[11];

(ix) Talent scouts, not practice scouts: talents are real [12];

(x) Creativity is an important factor over the practice [13];

(xi) The inborn talent yes, does exist [14].

Taking into account the large range of disperse and not coherent opinions on this topic, in order to try to clarify some fundamental aspects concerning it, in this paper it is discussed the transgenerational transmission of information and the innate predispositions from the perspective of the recently elaborated Informational Model of Consciousness [15-18], which explains the nature and structure of consciousness [17,19-21], its properties [22-24] and the neuro relations with the body [24-26], allowing to develop useful applications [27-29].

The Informational Model of Consciousness: Basic Structure and Transmission of Information

In the left side of (Figure 1) it is shown schematically the structure of consciousness, based on the concept of information. To understand this concept in its various forms, we have to point out that information could be interpreted as an informal property, like that received from the mass media or surrounding sources by our own senses (sight, hearing, smell, taste, touch), or intrinsic matter-related information, intervening into the intimate microscopic structure of matter [16,18]. A much more quantity of information is integrated into the living structures, in the human body, where practically all the constituting components-the living cells-communicate with themselves and with the others in a continuous and intimate manner [15]. For this reason, we may define the human body as an informed matter structure. Therefore, consciousness could be regarded as the representation/reflection in the mind of the functionality of specific informational systems which refer to:

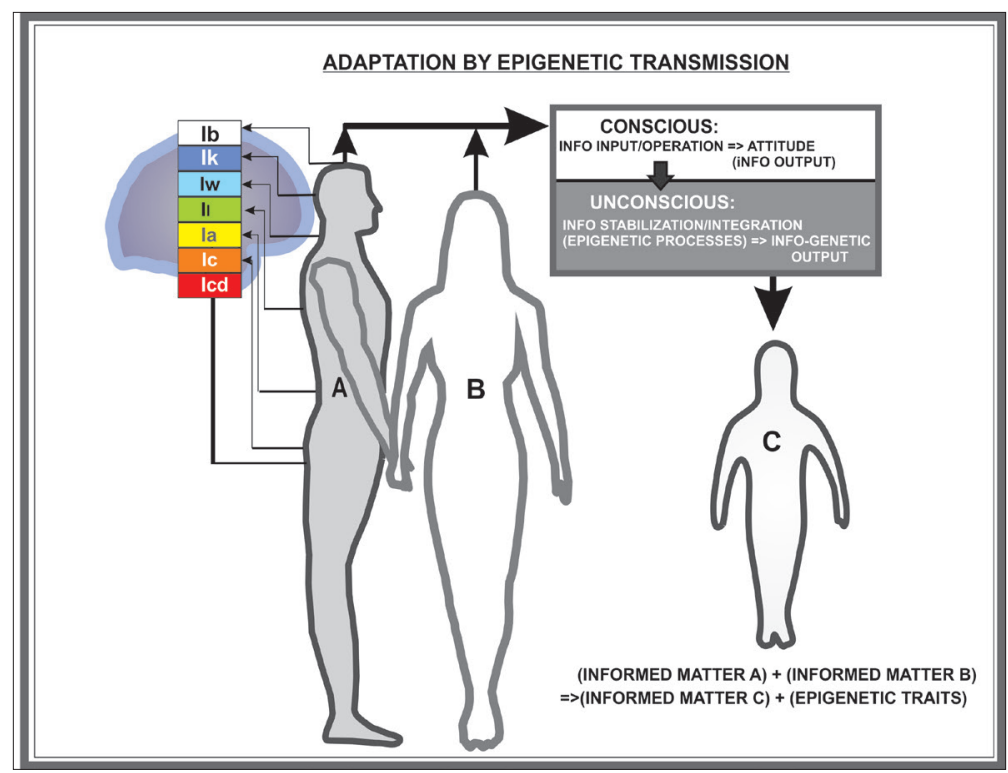

Figure 1: Schematic representation of the components of the Informational Model of Consciousness, their connections with the corresponding body execution elements and the transgenerational transmission of information. 
i. Accumulation of information (memory), marked suggestively in Figure 1 as the informational center I know (I k);

ii. Operation with information and decision, marked as the center I want (I w);

iii. Emotional activities, defined as I love (II), love being the most representative of them;

iv. Metabolic activities for body maintenance, connected to nourishment matter, marked as the informational center I am (Ia), indicating the self-status, power and health;

v. The living/genetic creation assuring the reproduction, reflected in consciousness as the informational center Icreate (Ic);

vi. Info-genetic generation, transmitting the genetic characteristics from the parents (informed matter A and B in Figure 1) to the new body (informed matter $C$ ), and represented by the informational center suggestively called I created (I cd);

vii. Info-connection activities, consisting especially in the control of the certainty/uncertainty balance, which reinforces the confidence, trust and equilibrium, the power to choose and believe, therefore defined as the informational center Ibelive (Ib).

Each of informational system relates to specific areas of the brain and with the specific execution system by means of the neuro cord and neuro derivations to the body organs [26], schematically represented in Figure 1 left side. The center $\mathrm{Ib}$ and its connections explain the near-death phenomena (ex-corporal view, "tunnel" crossing, peace and light feeling, virtual meeting with the members of family who are no longer) during the clinical arrest of the heart and cease of the brain activity [22], the special properties of the mind like premonition, clairvoyance, telepathy and psychokinetic phenomena [19,23], religious and mystic experience [15,21], and even the possibility of an after-life existence of consciousness as an independent entity [24]. During the day by day activities, the main role of this center is to function as a life "navigation lighthouse" and "helm", assuring the right/believed way to the life objectives and mobilizes, maintains and focuses the internal forces to the fulfilling of these objectives. In informational terms, this center represents an anti-entropic pole of information, because I believe signifies the selection from an uncertainty range of informational possibilities (which expresses actually the entropy of such an informational field) a certain information, which induces in consciousness and body security, trust and confidence, helping in this way the living prerogatives. Therefore, this center could be also regarded as an anti-entropic pole of the organism, assisting and supporting the living processes.

The memory (Ik) accumulates not only the life experience, as informational and emotional remembrances [17], but also the decision criteria, according to the mentality, especially acquired and consolidated during the childhood [20]. During the life span, this accumulation allows to attain the wisdom in the advanced age, when new criteria are added from the analysis of the various events of the life experience, from the selection between "Good" and "Bad" induced results. The life itself is a base of continuous learning for adaptation.

The adaptation process is managed by the operational/ decisional center of information Iw. Although the entire information is memorized as a life experience by Ik, only Iw may select and activate only the necessary information which serves as a basis of informational analysis and decisional process. The qualities of this center could be associated with the intelligence, speed of reaction/ operation and the correctness of the solution. However, this center operates based on decision criteria, most of them acquired in the familial ambient, enriched with that acquired during the life experience, including the professional and social one [20]. Besides the quantity and quality of the acquired and stoked information (Ik), emotions (Il), familial and social relations (Ic), inherited affinities (Icd), certitude/belief (Ibelieve) and the personal power state (Ia) contribute also to the final decision, so this will depends actually on signals received from the all other centers. The main result of the decision center Iw is the Attitude, which is the informational output of the conscious component of the informational system of the human body, necessary for immediate and/or medium-term adaptation to the environmental requirements.

Emotions, activated and managed by Il, could play an important role in decision. Emotions represents information stoked also in memory (Ik), but these are reactions of the body to the primary information, received or activated by Iw from the data library Ik. The emotions could be activated automatically, as a defense reaction, so are triggered very quickly by the alarm system of the brain (amygdala) or could be the result of some slower chemicaltype processes [30]. We defined such emotions based on chemical agents as emo-states and could be triggered consciously by the memorized or acquired information [15]. From this point of view, while the activities of Ik, Iw are conscious, the activity of Il is rather combined between conscious and unconscious one. The practice for a long time of the same type of emotions could create an emodependence on such induced states, with favorable consequences or not, so the activity of Il should be closely observed and suitably controlled [20].

As emotions can be therefore still controlled by conscious mind, the activities of Ik, Iw and Il represent the so-called operative informational system, which basically determines the reaction to information and is expressed by Attitude (Figure 1, upper right side). The center Ia reflects the status of the autonomic and automatic metabolic processes, because of the body connection to the external necessary matter (foods, air, water). Besides the common signals detected by the internal sensors and transmitted to the brain referring to the nourishment needs, like hungry and thirsty, this center vigils also the general situation of the body itself, the health, power and appropriate energy. By means of the autonomic execution systems managed by the programmed informational center of the brain, the absorbed matter is converted into the useful nutrients for the cells, assuring energy and substitution or reproduction materials for the body. The self-appreciation and selfesteem are close components of the characteristic range of qualities of this center. 
The center lc is closely linked to the relations with the partner of life, with the members of own formed family and with the society. The high objective of life is to create offspring and to form and educate the new generation, assuring in this way the species continuity and survival. The information is transmitted as a genetic output (Figure 1, right side), which is a codified ("imprinted)" form of living informed matter, participating together with the complementary genetic information of the partner to the creation of the new member of the family. The relative relations between generations are very close, as could be expressed in popular terms by consanguinity. Such term is a result of ancient multigenerational observations, claiming that the constituent common genetic information is a basic support of common physical and psychical features. The information baggage genetically transmitted, and the information transmitted by education especially within the first years of life, when the nervous system of the child is under intensive structuration, is fundamental for the formation of mentality of the new individual.

Mentality includes a set of guiding rules and automatic chain-thinking prototypes inherited from parents and acquired by education, which states the fixing points and criteria of informational processing for own decision making. The capacity of conscious acquirement and processing of information is limited, so many of our typical behavioral manifestations are integrated into the automatic informational system, as less thinking-effort consuming [27]. The parent attitude on the children comportment is therefore of great importance during this stage and not only. Within the confrontation with the life problems, the silent voice of the parents and their advising recommendations accompanies everywhere and whenever the future adolescent and adult, even toward the ancient age. This close relation is reflected within the informational activity of the center Icd of the new generation ( $\mathrm{C}$ in Figure 1, right downside). Within Icd are manifested the inherited properties of the new nascent child and future adult. The genetic information is transmitted by the genetic system and is manifested both in the similarity of the body and psychological features. During the life span this center manages also the typical characteristics of the age, from infancy to convenience.

As conscious cannot have access and interventions in the activities of the informational systems supporting the centers Ia, Ic and Icd, which are based, as it commented above on intimate molecular processes, the sum of these systems forms the unconscious component of the informational system of the human body (marked in obscure color in the right side of the Figure 1). The info output of the unconscious system is represented by the genetic transmission of information. The information in this case is supported by genetic matter, so according to the Informational Model of Consciousness, the transfer of information is done as a matter-related information.

The Silent Voice of Those Who Are no Longer: Transgenerational Transmission of Information

We name aptitude a series of manifested easiness with which somebody can think or make something specific. This is noted by the person himself or herself, and by the others. Such a behavior it is not qualified as a "hard" or difficult task. That is because the silent voice of the inherited properties is manifesting on this way. The affinity for a certain type of activity, expressed by the easy acceptance and ability to reproduce it without efforts is also due to the inherited features. On an increasing amplitude scale of the inherited qualities could be placed the tendency, affinity, propensity, predisposition, aptitude, vocation and talent. The silent voice of those who are no longer is manifested in various tones, registries and intensities; natural grace, innate gift, ability, skill, aptness, flair, knack, brilliance, belong to such a category.

The large range of existing words and concepts attributed to the inheritance qualities reflects the fine, graduated tuning scale of such qualities and the large recognition of the info-transference phenomenon from the predecessors to the offspring. If we want to refer to the info-material support of such inherited transference and to the related mechanisms, we should look no further than the early 90's, when the new branch of genetics science, known now as epigenetics, started to flourish. Within the Informational Model of Consciousness, it was recently shown that the specific epigenetics mechanisms, allowing the intimate transference of information inside and between cells can be interpreted in terms of information science, by a process of embodiment/disembodiment of information [15]. Epigenetics studies the mechanisms which determines the acquisition of information at cellular level, induced by the environmental cues and stress. By stress we have to understand the factors situated outside of the commonly accepted range in the natural living ambient, and could be referred to whether, foods, or any other factor which can intervene to change the individual or group behavior, submitted under such circumstances to an adaptation process, but without change the genetic configuration.

One effect of this epigenetic process could be the transmission of the acquired traits to the next generation or generations, if this stress continues to persist (Figure 1). Such factors like drug or alcohol addiction were demonstrated to have influence on the next generation, specifically on the female (mother) line [31]. Epigenetics processes are also implicated in memory formation [32]. In a recent study it was shown that parents' stressful experiences can influence an offspring's vulnerability to many pathological conditions, including psychopathologies, and their effects may even endure for several generations. In this recent study it was confirmed that the main types of such epigenetic dynamic processes could be direct, when the changes occur during the lifespan of an individual due to a direct exposure to the environment experience, or indirect, both when it concerns changes that occur inside of the womb due to events during gestation and changes that affect the individual's predecessors (parents, grandparents, etc.), due to events that occur even long before conception and that are somehow (e.g., through gametes, the intrauterine environment setting) transmitted across generations [33].

The intimate epigenetic process consists in three main steps as follows. During the first step, the signal which originates the interaction of the perceived information with the body itself is the 
"epigenator", which can be molecules of the cell, "informed" by an info-embodiment process $[15,27]$ and this signal triggers a cascade of cell molecular signal response, if the epigenetic is sufficiently persistent. Therefore, a repetitive and/or intensive signal is an imprescriptible condition that the epigenetic process should be initiated. In terms of epigenetics, the cell response signal is the "initiator", which acts on the chromosomes, composed by DNA (deoxyribonucleic acid 30-40\%), RNA (ribonucleic acid 1-10\%), and histones (50-60\%), which are some proteins anchoring the DNA helix structure. The proportion of these components varies not only for different species and for various tissues of the same organism, but even in the same cell, depending on the various stages of the cell cycle [15]. Finally, the epigenetic "maintainer" signal conserves the stable embodied information which will be transmitted to the daughter cells. In terms of the Informational Model of Consciousness, the insistent repetition of an information from the environment is a key factor for the epigenetic processes, allowing the transgenerational transmission of the traits. The repetition and/or intensive exposure to the epigenetic signal allows the initial informational signal to be transferred from the short-term memory to the long-term memory and from here to the memory of the skills, a complex and complete set of info-abilities automatically triggered [27], as it is for instance the playing an instrument or driving a bike [15].

The practice is therefore an important component of the performance, because by its natural form of working, the brain asks repetitive steps for a stable info-integration into the cells. However, what are the "epigenetics" and info-"initiators" of such a process? Who calls a child or young person to dedicate his time, energy and internal forces to a certain type of activity? This is the silent voice of those who are no longer (Figure 1, right lower side-transmitted epigenetic traits). As external observer, we call the manifestation of this voice affinity, propensity, predisposition, aptitude, vocation or talent. As personal self-own observers, we follow the calling of this voice, which indicates us that a certain activity is easy and pleasant to be done, stimulating the creativity, dedication and perseverance in practice and/or in a deep and profound study. This depends of course on the intensity of this voice, which means the intensity which the transgenerational signal was epigenetically transmitted by Icd. If this signal is weak and fragile, as often could happen at the children, whose power of Ia, Il and Ib is still low, it is necessary of course a stimulating impulse and encouragement from the side of parents, educators or instructors. If this signal is sufficiently high to be self-detected, the personal center Ib has a sufficient force to maintain the "navigation helm" on the right direction, assuring the confidence, trust and tenacity for the fulfilling of the final objective: the talent full manifestation.

\section{Conclusion}

It was shown that the old controversy on "nature or nurture" problem referring specifically to the inherited properties of the human like predispositions and talent with respect to the acquired ones by practice, can be approached in terms of transgenerational transmission of information, from the perspective of the
Informational Model of Consciousness. This model reveals the existence of seven informational centers with specific activities, each of them with distinct tasks but interacting and collaborating each other's for suitable adaptation to the environmental cues and stressors. The center Ik is the memory, Iw the operational decision, Il the emotion center, Ia self-status center reflecting the state of the metabolism operations, Ic the genetic transmission, Icd the info - genetic generator and Ib the anti-entropic center, assisting the life prerogatives, because it reduces to certainty the large range of uncertainty possibilities. As the Informational Model of Consciousness is able to describe in terms of information the intimate cellular transmission by mechanisms of embodiment/ disembodiment of information, it was shown that the epigenetic processes acts as convertors of the received information from the environment into the matter-related epigenetic information, transmissible by Ic to the offspring as genetic output.

The center Icd reflects the inherited physiological and psychological properties, particularly the affinity, propensity, predisposition, aptitude, vocation and talent. The large spectrum of the observed and defined inherited qualities shows the fine degree of variety and of signal intensity with which the silent voice of those who are no longer, the family predecessors, may express their acquired traits to the present generation. This signal is manifested to the own person and to the external observers. The practice is an important requirement that the talent become an evidence for the external observers, because the acquirement of stable information both by nervous system and epigenetic processes is based on repetitive and intensive training. However, the various self-observed internal signals coming from the precedent generations marking predispositions and affinities are easily detected, indicating that certain activities are facile and pleasant to be done. Just these signals are the predispositions, aptitudes, affinities and the entire range of the inherited tendency qualities. If the voice of those who are no longer is low at the children, then encouragement and stimulation impulses from the side of parents, teachers and instructors should be expressed. If these voices are sufficiently high, then the child or the young person himself or herself will meet the force (Ia), mental capacity (Ik, Iw) and tenacity (Iw), attachment feeling (Il), trust and confidence (Ib) to follow the voice calling (Icd) to attain the performance lciated by the external observers as talent.

\section{Acknowledgment}

As a continuity of life and information, the author dedicates this work to his son Adrian Gaiseanu and his family, together with special appreciations for his dedicated work and results. To his daughter Ana with special appreciations and to all members of family who appreciate and love him.

In the memory of his eminent and exemplar parents, the distinguished (Magna cum Laude) Professor Emanoil Gaiseanu and distinguished (Emeritus) Professor Florica Gaiseanu and of all Members of Family who were part of his life: his brother Professor Constantin Gaiseanu, Fam. Popescu, Plopeanu, Potlogeanu, whose silent voices are still intimately listened. 


\section{References}

1. Howe M, Davidson J, Sloboda J (1998) Innate talents: Reality or myth? Behav Brain Sci 21(3): 399-407.

2. Baltes $P$ (1998) Testing the limits of the ontogenetic sources of talent and excellence. Behavioral and Brain sciences 21(3): 407-408.

3. Bronfenbrenner U, Ceci S (1998) Could the answer be talent? Behavioral and Brain Sciences 21(3): 409-410.

4. Charness N (1998) Explaining exceptional performance: Constituent abilities and touchstone phenomena. Behavioral and Brain Sciences 21(3): 410-411.

5. Csikszentmihalyi M (1998) Fruitless polarities. Behavioral and Brain Sciences 21(3): 411.

6. Detterman D, Gabriel L, Ruthsatz J (1998) Absurd environmentalism. Behavioral and Brain Sciences 21(3): 411-412.

7. Eisenberger R (1998) Achievement: The importance of industriousness. Behavioral and Brain Sciences 21(3): 412-413.

8. Ericsson KA (1998) Basic capacities can be modified or circumvented by deliberate practice: A rejection of talent accounts of expert performance. Behavioral and Brain Sciences 21(3): 413-414.

9. Feldman D, Katzir T (1998) Natural talents: An argument for the extremes. Behavioral and Brain Sciences 21(3): 414.

10. Heller K, Ziegler A (1998) Experience is no improvement over talent. Behavioral and Brain Sciences 21(3): 417-418.

11. Humphreys L (1998) General intelligence is central to many forms of talent. Behavioral and Brain Sciences 21(3): 418.

12. Rutter M (1998) Talent scouts, not practice scouts: Talents are real Behavioral and Brain Sciences 21(3): 421-422.

13. Weisberg R (1998) Creativity and practice. Behavioral and Brain Sciences 21(3): 429-430.

14. Freeman J (1998) Inborn talent exists. Behavioral and Brain Sciences 21(3): 415 .

15. Gaiseanu F (2019) The informational model of consciousness: Mechanisms of embodiment/disembodiment of information. NeuroQuantology 17(4): 1-17.

16. Gaiseanu F (2018) Information: from philosophic to physics concepts for informational modeling of consciousness. Philosophy Study 8(8): 368-382.

17. Gaiseanu F (2019) Destiny or free will decision? A life overview from the perspective of an informational modeling of consciousness part I: information, consciousness and life cycle. Gerontology \& Geriatric Studies 4(3): 1-7.

18. Gaiseanu F (2019) Informational model of consciousness: from philosophic concepts to an information science of consciousness. Philosophy Study 9(4): 181-196.
19. Gaiseanu F. Consciousness as Informational System of the Human Body. Consciousness and Life Physics, Cosmology and Astrophysics Journal 2016a; 16(1): 14-25.

20. Gaiseanu F (2018) Destiny or Free Will Decision? A Life Overview From the Perspective of an Informational Modeling of Consciousness Part II: Attitude and Decision Criteria, Free Will and Destiny. Gerontology \& Geriatric Studies 4(1): 1-7.

21. Gaiseanu F (2019) Human/humanity, consciousness and universe: Informational relation. NeuroQuantology 17(5): 60-70.

22. Gaiseanu F (2017) Quantum-assisted process of disembody under near-death conditions: an informational-field support model. NeuroQuantology 15(1): 10-17.

23. Gaiseanu F (2017) An Information based model of consciousness fully explaining the mind normal/paranormal properties. NeuroQuantology 15(2): 132-140.

24. Gaiseanu F (2018) Near-death experiences and immortality from the perspective of an informational modeling of consciousness. Gerontology \& Geriatric Studies 2(3): 1-4

25. Gaiseanu F (2019) Informational mode of the brain operation and consciousness as an informational related system. Archives in Biomedical Engineering \& Biotechnology 1(5): 1-7.

26. Gaiseanu F (2019) Informational neuro-connections of the brain with the body supporting the informational model of consciousness. Archives in Neurology \& Neuroscience 4(1): 1-6.

27. Gaiseanu F (2019) Epigenetic information-body interaction and information-assisted evolution from the perspective of the informational model of consciousness. Archives in Engineering \& Biotechnology 2(2): $1-6$

28. Gaiseanu F (2029) Language patterns and cognitive-sentient reality: Certainty/uncertainty in cognitive-sentient exploration of reality. In: Stephen BS (Ed.), Media Models to Foster Collective Human Coherence in the psychecology, IGI Global, USA.

29. Gaiseanu F, Graur A (2018) Cognitive centers related attitude: application for an iterative evaluation method in music-based therapy process. In: Stuart Hameroff (Ed.), Conference on Science of Consciousness, ArizonaCenter for Consciousness Studies, Arizona, USA.

30. Dispenza D (2007) Evolve your brain: The science of changing your mind. FL: Health Communications Inc, Deerfield Beach, Florida.

31. Goodman HS, Gotlib HI (1999) Risk for psychopathology in the children of depressed mothers: A developmental model for understanding mechanisms of transmission. Psychol Rev 106(3): 458-490.

32. Levenson JM, Sweatt JD (2005) Epigenetic mechanisms in memory formation. Nat Rev Neurosci 6(2): 108-118.

33. Lacal I, Ventura R (208) Epigenetic inheritance: Concepts, mechanisms and perspectives. Front Mol Neurosci 11: 292. 